\title{
Beam heat load and pressure rise in a cold vacuum chamber
}

\author{
S. Casalbuoni, * A. Grau, M. Hagelstein, and R. Rossmanith \\ Institute for Synchrotron Radiation, Research Center Karlsruhe, P.O. Box 3640, D-76021 Karlsruhe, Germany
}

\section{F. Zimmermann}

CERN, AB Department, 1211 Geneva 23, Switzerland

B. Kostka, E. Mashkina, and E. Steffens

Physics Institute II, Friedrich Alexander University of Erlangen-Nürnberg, Germany

A. Bernhard, D. Wollmann, and T. Baumbach

Laboratory for Application of Synchrotron Radiation, University of Karlsruhe, Germany

(Received 13 June 2007; published 20 September 2007)

\begin{abstract}
The beam heat load and the pressure in the vacuum chamber of the cold bore superconducting undulator installed at ANKA (ANgstrom source KArlsruhe) have been monitored for almost two years. Possible sources of the observed heat load could be synchrotron radiation from upstream magnets, image currents, electron and ion bombardment. In this paper, the various possible contributions to the heat load are discussed and compared with experimental results. The dynamic pressure increases nonlinearly with the average beam current. The current where it assumes a maximum varies both with the bunch intensity and with the initial vacuum pressure. A correlation between the heat load and the dynamic pressure has been observed. This study suggests that electron bombardment could explain the beam heat load and pressure rise observed for a bunch length of $10 \mathrm{~mm}$.
\end{abstract}

DOI: 10.1103/PhysRevSTAB.10.093202

PACS numbers: 29.27.- a, 41.75.Ht, 29.20.Dh, 84.71.Ba

\section{INTRODUCTION}

Superconducting undulators have higher fields for a given gap and period length compared with permanent magnet undulators. This technological solution is very interesting for synchrotron light sources since it permits to increase the brilliance [1,2] at relatively low costs. Superconducting undulator technology has also recently been acknowledged by the high energy particle physics community to be a promising solution for the CLIC damping-ring wiggler [3] and for the ILC positron source [4]. However, superconducting undulator technology is not yet mature. One of the key issues is the understanding of the beam heat load to the cold vacuum chamber. In this paper we present beam heat load measurements performed at the synchrotron light source ANKA (ANgstrom source KArlsruhe) in the superconducting cold bore undulator operating in the ring since March 2005.

\section{EXPERIMENTAL SETUP}

ANKA is an electron storage ring used as a synchrotron facility [5]. The maximum achievable energy is $2.5 \mathrm{GeV}$ and the maximum current is $200 \mathrm{~mA}$. The revolution time is $T_{r}=368 \mathrm{~ns}$ and the machine is normally operated with two trains, each composed of 32 bunches. The bunches are separated by 2 ns. The cold bore superconducting undulator built by ACCEL Instruments $\mathrm{GmbH}$, Bergisch

\footnotetext{
*Corresponding author: sara.casalbuoni@iss.fzk.de
}

Gladbach, Germany [6], is installed in one of the four straight sections of the ring, see Fig. 1; the rest of the ring is at room temperature. The vacuum chambers of the warm part of ANKA have been baked before installation at $200^{\circ} \mathrm{C}$ for 48 hours and vented with nitrogen. The storage ring compatible cryostat is shown in Fig. 2. The system is cryogen free and is cooled by three Sumitomo cryocoolers

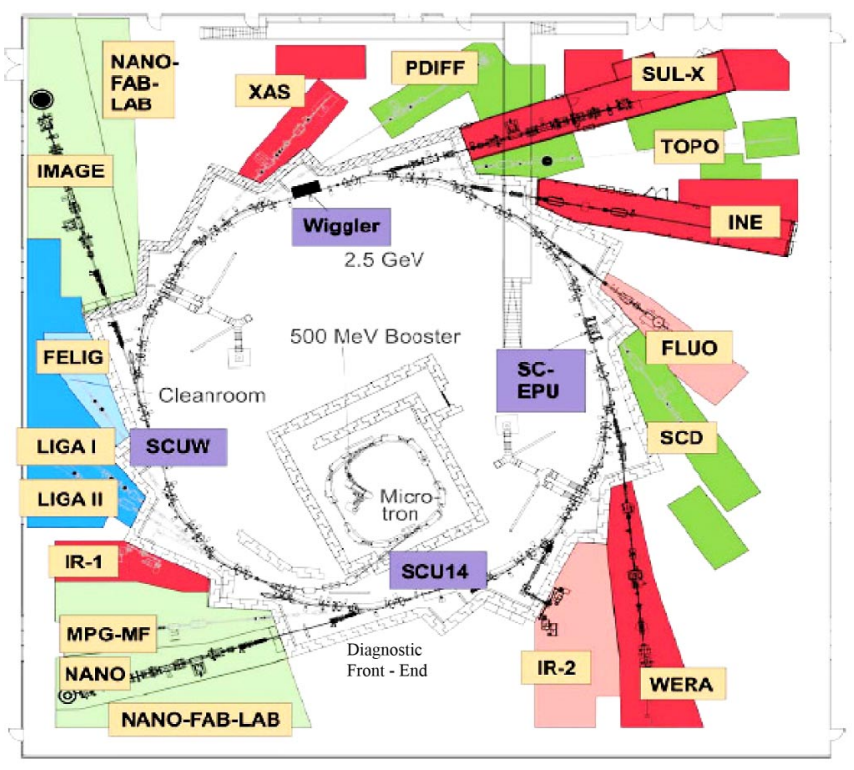

FIG. 1. (Color) The synchrotron radiation facility ANKA. The superconducting undulator is labeled SCU14. 


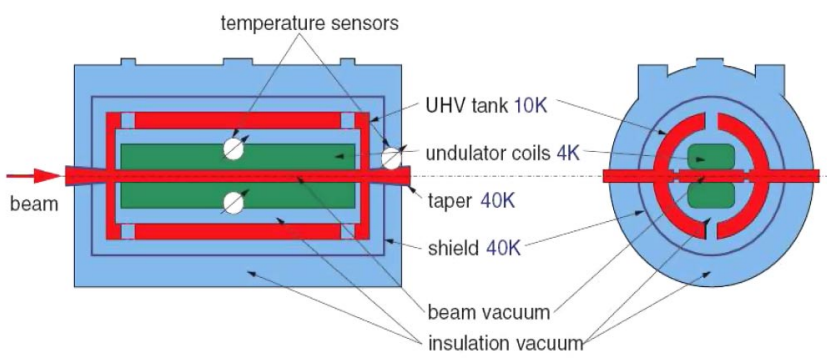

FIG. 2. (Color) Schematic layout of the vacuum system of the superconducting undulator and the position of the temperature sensors.

(RDK-408D @ 50 Hz) [7]: two of them cool the coils to about $4 \mathrm{~K}$ and one the ultrahigh vacuum (UHV) tank, which is at $10 \mathrm{~K}$ and protects the coils from the external thermal radiation. The cryostat consists of two separated vacuum systems for the cold mass: a UHV system for the beam and an insulation vacuum system for the coils and the rest of the cold mass. The pressure of the two vacua are monitored by pressure gauges at room temperature. A $300 \mu \mathrm{m}$ stainless steel foil coated with $30 \mu \mathrm{m}$ of copper is placed between the cold mass and the beam vacuum. A taper system connects the normal beam pipe with the cold mass and has two functions: (1) smooth transition for wake fields, (2) thermal transition between the cold bore at $4 \mathrm{~K}$ and the beam pipe at room temperature. Several temperature sensors are placed on the different elements: coils, UHV tank, taper entrance, taper exit, etc. The undulator can be operated with different gap sizes: 16,12 , and $8 \mathrm{~mm}$, and it can be opened to $29 \mathrm{~mm}$ without current in the coils during injection. Some of the parameters of the electron beam, the storage ring, and the superconducting undulator are summarized in Table I. In order to protect the undulator from the synchrotron radiation emitted by the upstream

TABLE I. Electron beam, storage ring, and superconducting undulator parameters.

\begin{tabular}{lc}
\hline \hline Electron beam & \\
\hline$E_{\max }$ & $2.5 \mathrm{GeV}$ \\
$I_{\max }$ & $200 \mathrm{~mA}$ \\
\hline Storage ring & $110.4 \mathrm{~m}$ \\
\hline Circumference & $499.66 \mathrm{MHz}$ \\
Cavity frequency & $368 \mathrm{~ns}$ \\
$T_{\text {rev }}$ & \\
\hline Undulator & $1.4 \mathrm{~m}$ \\
\hline Length & 100 \\
Periods & $8,12,16 \mathrm{~mm}$ \\
Magnetic gap & $29 \mathrm{~mm}$ \\
Beam stay clear gap & $0.8 \mathrm{~T}$ \\
Maximum magnetic field at $8 \mathrm{~mm}$ gap
\end{tabular}

magnets, a collimator system is located at about $1 \mathrm{~m}$ from the entry point of the undulator [8].

\section{POSSIBLE HEAT LOAD SOURCES}

Possible heating mechanisms are: (1) synchrotron radiation from upstream magnets, (2) high frequency image currents on the cold surface also called resistive wall heating, (3) ions and electrons accelerated to the walls by the transverse field of the ultrarelativistic beam. The heat load sources depend differently on beam parameters such as beam energy $E$, average beam current $I$, total number of bunches $M$, and the bunch length $\sigma_{z}$. Measuring the beam heat load varying the above-mentioned parameters allows to distinguish between the different heating mechanisms.

\section{A. Heat load from synchrotron radiation}

The power of the synchrotron radiation emitted from the upstream bending magnet hitting the upper and lower surfaces of the vertical gap of the undulator is [9]

$P_{\mathrm{syn}}=2 P_{0} \frac{21}{32} \int_{\psi_{0}}^{\psi_{1}} \frac{\gamma}{\left(1+\gamma^{2} \psi^{2}\right)^{5 / 2}}\left[1+\frac{5}{7} \frac{\gamma^{2} \psi^{2}}{\left(1+\gamma^{2} \psi^{2}\right)}\right] d \psi$,

where $\psi_{0}$ and $\psi_{1}$ are the lower and upper values of $\psi$ indicated in Fig. 3,

$$
\gamma=E / m_{e} c^{2}, \quad P_{0}=\frac{e I \gamma^{4}}{6 \pi \epsilon_{0} \rho},
$$

$e$ is the electron charge, $I$ is the average beam current, $\epsilon_{0}$ is the vacuum permittivity, $E$ is the beam energy, $\rho$ is the radius of curvature of the electron trajectory in the bending magnet, $m_{e}$ is the electron mass, and $c$ is the speed of light. The factor 2 in front of the integral takes into account of the upper and lower surfaces of the vacuum chamber of the undulator (see Fig. 3). The power dissipated in the ANKA superconducting undulator assuming perfect alignment, a current of $100 \mathrm{~mA}$, and a gap of $8 \mathrm{~mm}$ is $63 \mathrm{~mW}$ [8].

\section{B. Heat load from resistive wall effect}

The resistive wall heating losses per unit length can be calculated by [10]

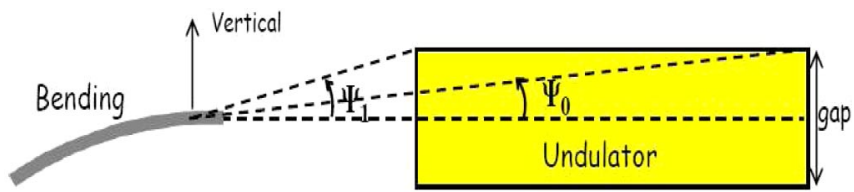

FIG. 3. (Color) Scheme of the synchrotron radiation from the upstream bending magnet hitting the upper and lower surfaces of the vacuum chamber of the superconducting undulator. 


$$
P_{\mathrm{RWH}}=\frac{I^{2}}{M f_{0} \pi^{2} g} \int_{0}^{\infty} S^{2}(\omega) R_{\mathrm{surf}}(\omega) d \omega[\mathrm{W} / \mathrm{m}]
$$

where $I$ is the average beam current, $M$ is the number of bunches, $f_{0}$ the revolution frequency, and $g$ is the gap (an equivalent formula is given in Ref. [9]). $S(\omega)$ is the bunch spectrum. Assuming a bunch with Gaussian shape and length $\sigma_{z}$,

$$
S(\omega)=e^{-\left(\sigma_{z}^{2} \omega^{2}\right) /\left(2 c^{2}\right)} .
$$

$R_{\text {surf }}(\omega)$ is the surface resistance. The vacuum chamber is made of a $300 \mu \mathrm{m}$ layer of stainless steel electroplated with $30 \mu \mathrm{m}$ of copper. Electroplated copper has usually a residual resistivity ratio (RRR) in the range between 10 and 100 [9]. For copper at low temperatures and RRR $>7$ the anomalous skin effect [9-11] has to be considered:

$$
R_{\text {surf }}(\omega)=R_{\infty}(\omega)\left(1+1.157 \zeta^{-0.276}\right), \quad \text { for } \zeta \geq 3
$$

with

$$
\zeta=\frac{3}{2}\left(\frac{\ell}{\delta(\omega)}\right)^{2}=\frac{3}{4} \mu_{r} \mu_{0} \sigma \omega \ell^{2},
$$

where $\ell$ is the electron mean free path in copper,

$$
\delta(\omega)=\sqrt{\frac{2}{\mu_{r} \mu_{0} \sigma \omega}}
$$

is the skin depth, $\mu_{r}$ the relative permeability, $\mu_{0}$ the vacuum permeability and $\sigma$ the electrical conductivity, and

$$
R_{\infty}(\omega)=\left(\frac{\sqrt{3}}{16 \pi} \frac{\ell}{\sigma}\left(\mu_{r} \mu_{0} \omega\right)^{2}\right)^{1 / 3}
$$

is the surface resistance in the limit of $\ell \gg \delta(\omega), \ell \rightarrow \infty$. For copper $\ell / \sigma=6.8 \times 10^{-16} \Omega \mathrm{m}^{2}$ [12] and the conductivity at room temperature is $\sigma=5.68 \times$ $10^{7} \Omega^{-1} \mathrm{~m}^{-1}$ [12]. Assuming $\mathrm{RRR}=100$, the electron mean-free path at $4.2 \mathrm{~K}$ is $\ell \simeq 4 \mu \mathrm{m}$. With $\mathrm{RRR}=100$, $I=100 \mathrm{~mA}$, a bunch length of $10 \mathrm{~mm}$, and a gap of $8 \mathrm{~mm}$, the resistive wall heating to the ANKA undulator is only $22 \mathrm{~mW}$ [8].

Synchrotron radiation losses increase linearly with the average beam current, while resistive wall heating losses scale with $I^{2} / M$ and depend strongly on the bunch length $\sigma_{z}$.

\section{Heat load from electron and ion bombardment}

A naive model of heat load due to electron and ion bomdardment is described below. A charged particle with mass $m$ and charge $q$ in the vacuum chamber can be accelerated by the transverse electric field carried by the ultrarelativistic bunch:

$$
E(r)=\frac{\lambda}{2 \pi \epsilon_{0} r},
$$

where $\lambda$ is the line charge of the bunch,

$$
\lambda=\frac{e N_{b}}{c \tau},
$$

and $\tau$ is the bunch duration and $N_{b}$ the particles per bunch

$$
N_{b}=\frac{I T_{r}}{e M}
$$

with $T_{r}$ the revolution time [13]. The momentum transfer by one bunch to a stationary electron at radial position $r$ is

$$
\Delta p=q E(r) \tau=\frac{q e N_{b}}{2 \pi \epsilon_{0} c r}
$$

and the corresponding energy increase

$$
\Delta W=\frac{\Delta p^{2}}{2 m}=\frac{\left(\frac{q e N_{b}}{2 \pi \epsilon_{0} c r}\right)^{2}}{2 m} .
$$

Roughly, the power is the energy gained by the charged particle $\Delta W$ times the number of charged particles accelerated to the wall per unit time $\dot{\Gamma}$ :

$$
P_{\mathrm{el}}=\Delta W \cdot \dot{\Gamma} .
$$

Since the energy $\Delta W$ is inversely proportional to the mass of the particle $m$, the ion contribution can be neglected, see Eq. (6).

A possible source of electrons is the condensed gas layer physisorbed on the surface (see Fig. 4). The gas layer is formed at low temperatures since energetic particles first hit the surface oxide layer of the vacuum chamber. The gases desorbed from the oxide layer then recondense on the surface so that the energetic particles will afterwards hit the physisorbed gas layer where the molecules are only loosely bound (binding energy few meV) by van der Waals forces. Since the molecules forming the condensed gas layer have already been desorbed, this phenomenon is usually referred to in the literature as "recycling" (see, for example, Ref. [15]).

Our vacuum chamber surface is very similar to the one of the LHC beam screen $(300 \mu \mathrm{m}$ stainless steel with $30 \mu \mathrm{m}$ of electroplated copper). For such a surface, the dominating desorbed gases are $\mathrm{H}_{2}, \mathrm{CH}_{4}, \mathrm{CO}, \mathrm{CO}_{2}$, and $\mathrm{H}_{2} \mathrm{O}$. Of these only $\mathrm{H}_{2}$ has a non-negligible vapor pressure at $4-20 \mathrm{~K}$. The plot in Fig. 5 shows the adsorption iso-

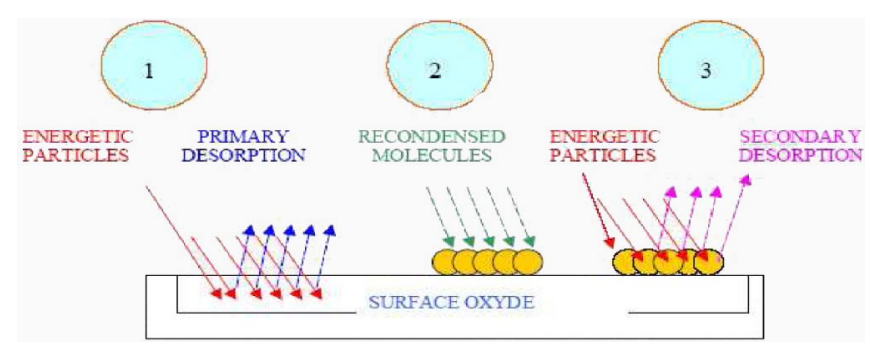

FIG. 4. (Color) Scheme of the desorption process on a cold surface. After Hilleret [14]. 


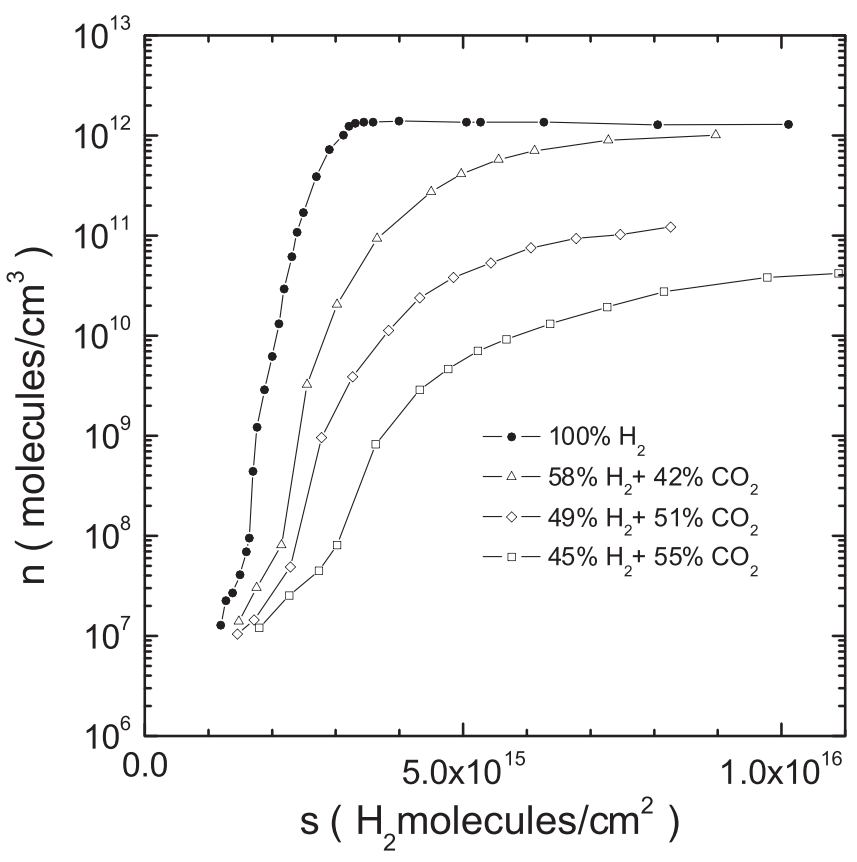

FIG. 5. Isotherms of coadsorption of $\mathrm{H}_{2}$ and $\mathrm{CO}_{2}$ on $\mathrm{Cu}$ plated stainless steel at $4.2 \mathrm{~K}$, plotted as a function of the surface density of $\mathrm{H}_{2}$ molecules. Courtesy of Wallén [16].

therm of $\mathrm{H}_{2}$ at $4.2 \mathrm{~K}$, that is the density of $\mathrm{H}_{2}$ molecules in gas form $n$, corresponding to the equilibrium pressure measured at room temperature $P_{\mathrm{UHV}}=n\left(\mathrm{~mol} / \mathrm{cm}^{3}\right)$. $4.9 \times 10^{-18}$ mbar [16] as a function of the $\mathrm{H}_{2}$ surface coverage $s$. The adsorption isotherm varies with many parameters as the molecular species present in the surface oxide, on the surface temperature, on the nature of the surface oxide, and on the gas composition inside the chamber. In the vacuum chamber of the superconducting undulator, the equilibrium pressure is about $(2-5) \times$

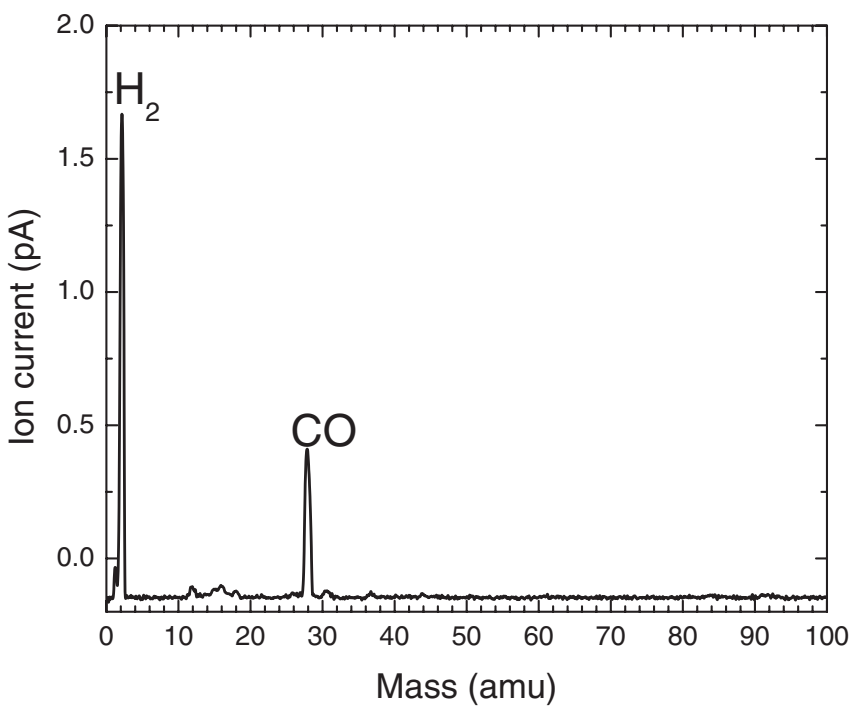

FIG. 6. Mass spectrum of the warm vacuum chamber just before the undulator with beam.
$10^{-11}$ mbar, which corresponds to $10^{15} \mathrm{H}_{2}$ molecules per $\mathrm{cm}^{2}$. Taking into account the geometry of the vacuum chamber, the number of $\mathrm{H}_{2}$ molecules on the surface and in the volume can be calculated. On the surface $N_{\text {Surf }} \approx$ $10^{17}$ and in the volume, considering $P_{\mathrm{UHV}}(300 \mathrm{~K})=$ $10^{-11}$ mbar, $N_{\mathrm{Vol}} \approx 5 \times 10^{10}$. On the surface there are more than $10^{6}$ times more molecules than in the volume: the surface is a huge electron reservoir. The mass spectrum of the warm vacuum chamber just before the undulator with beam shows only the $\mathrm{H}_{2}$ and $\mathrm{CO}$ lines, see Fig. 6. $\mathrm{CO}$ disappears when there is no beam: this is an indication of the dynamic of the desorption and recycling process discussed above. In the rest of the ring most of the time no $\mathrm{CO}$ is detected.

\section{MEASUREMENTS}

\section{A. Beam induced heat load during normal beam operation}

Figure 7 shows the average beam current, the beam energy, the UHV pressure [17], and the temperature of the coils as a function of time during user operation in a time range of 2 weeks. The increase in temperature of the coils is a measure of the deposited beam heat load. The calibration was done with a resistor in thermal contact with the coils. Thermal equilibrium is reached after two hours. The beam heat load to the coils is about $1 \mathrm{~W}$. The pressure increases after injection. The heat load (upper plot) and the pressure (lower plot) from different fills as a function of the beam current are shown in Fig. 8. The curves that can be visualized correspond to different fills. A correlation between the heat load and the pressure is observed (see inset of the upper plot). In the lower plot of Fig. 8 a nonlinear increase in the pressure is observed by increasing the beam current. A maximum of the pressure is observed for an

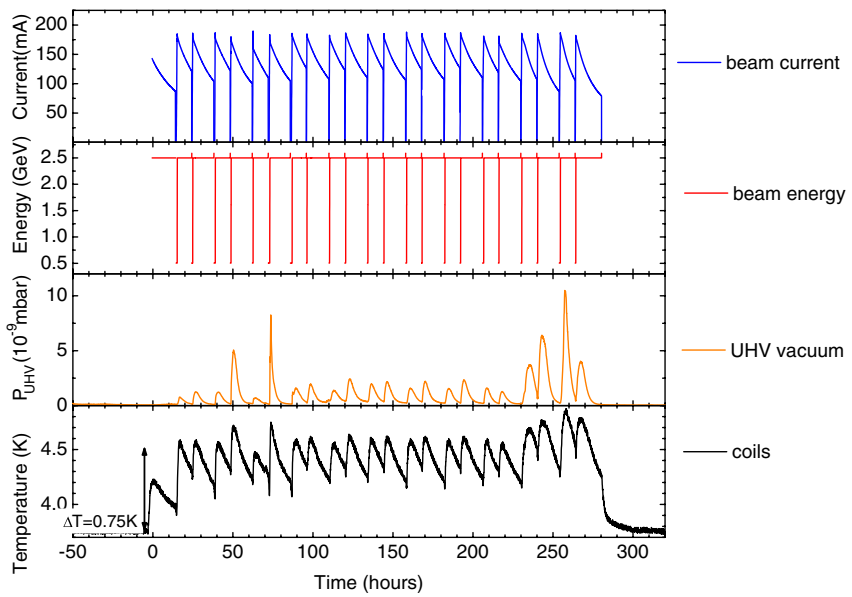

FIG. 7. (Color) Typical user operation run with open gap $(=29 \mathrm{~mm}$ ) and no current in the undulator. The beam current, the beam energy, the UHV pressure, and the temperature of the coils are reported as a function of time. 

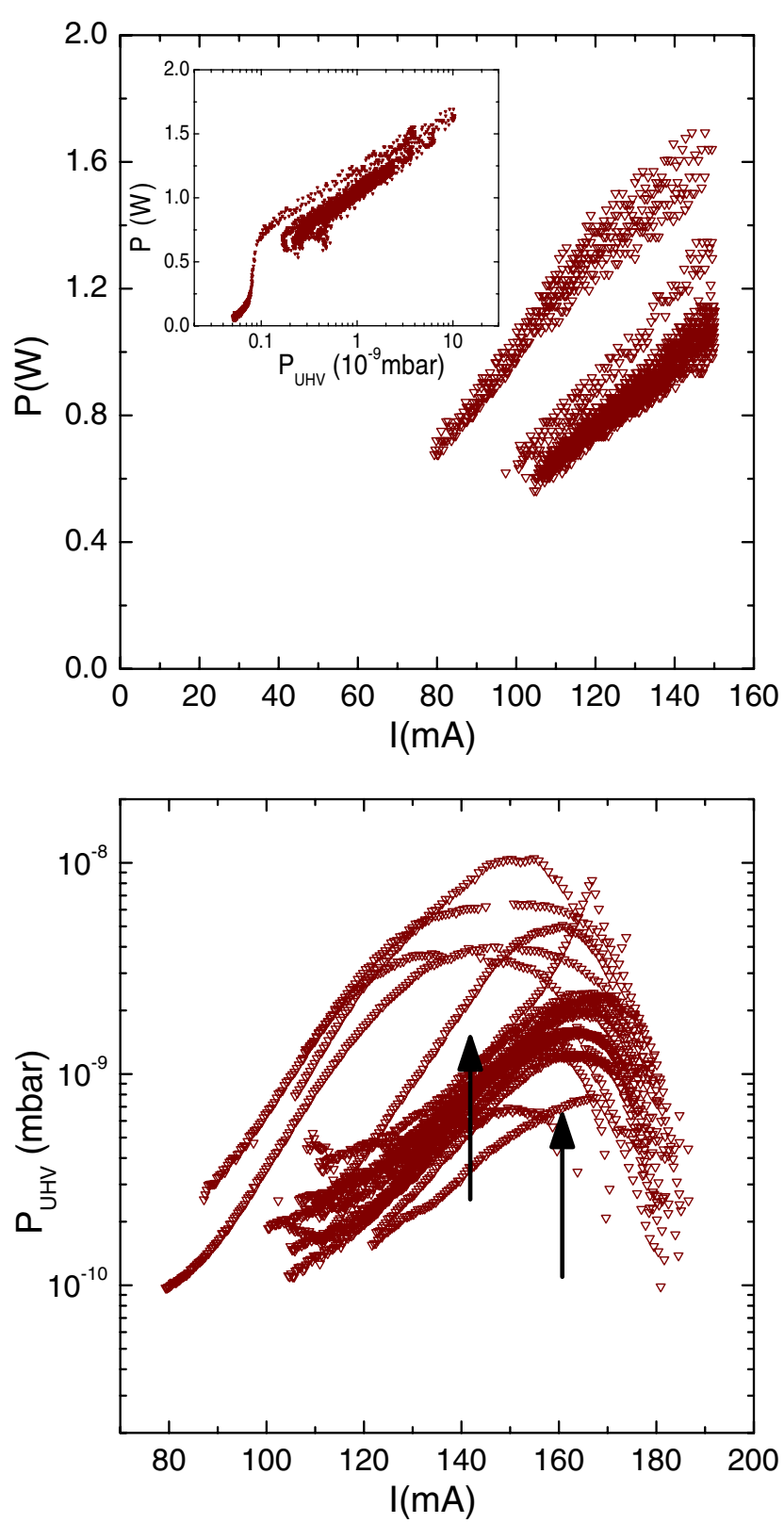

FIG. 8. (Color) Upper plot: the beam heat load from Fig. 7 is shown as a function of the average beam current $I$ and in the inset the beam heat load as a function of the UHV pressure. Lower plot: the UHV pressure from Fig. 7 is shown as a function of the average beam current $I$. The curves that can be visualized correspond to different fills. The arrows indicate the current where the peak pressure is observed.

average current of about $160 \mathrm{~mA}$. The peak shifts to lower currents $(140 \mathrm{~mA})$ when the pressure is higher. A similar pressure rise with current has been observed in the positron ring at the B factory PEP-II, a warm machine, for high currents and has been attributed to electron multipacting [18].

During the user operation in ANKA, a large variation of the heat load and of the UHV pressure is observed. Figure 9
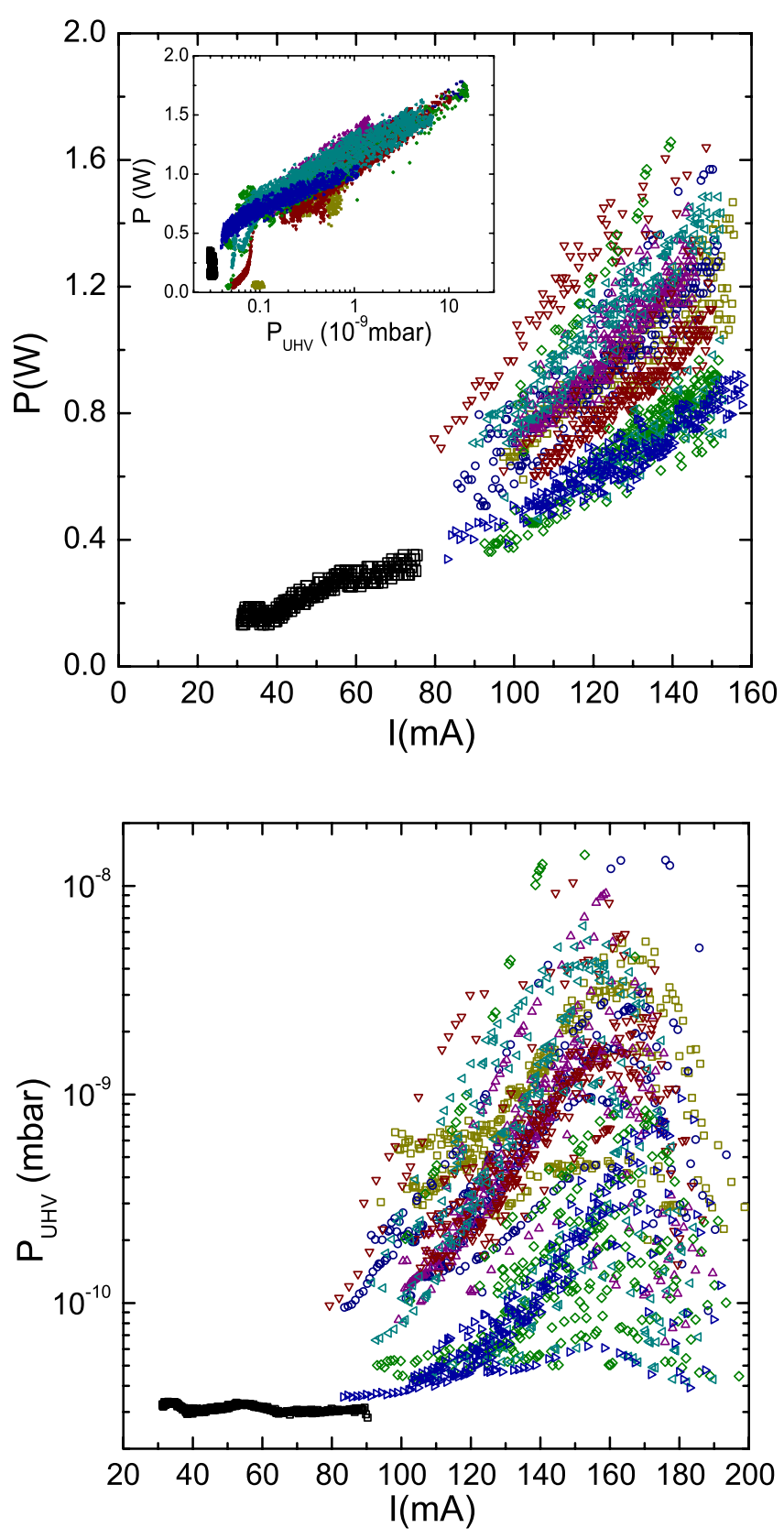

FIG. 9. (Color) Variation over half a year of the beam heat load (upper plot) and of the UHV pressure (lower plot) reported as a function of the average beam current. The different colors refer to different runs over a period of about two weeks. In the inset of the upper plot, the beam heat load is shown as a function of the UHV pressure. Beam parameters: $E=2.5 \mathrm{GeV}, I=$ 80-200 mA, two trains. Undulator parameters: gap $=29 \mathrm{~mm}$, undulator current $=0 \mathrm{~A}$.

shows the heat load (upper plot) and the UHV pressure (lower plot) as a function of the average beam current $I$ measured over half a year. In all cases the orbit is identical. The different colors refer to different runs over periods of about two weeks (similar to what is shown in Figs. 7 and 8). A low current run indicates the correct calibration of the 
beam heat load: the heat load goes to zero for low beam currents. In the inset of the upper plot of Fig. 9, the beam heat load is shown as a function of the UHV pressure. A correlation between the two measured quantities is observed: above a certain heat load and pressure the heat load increases by increasing the pressure, below it is independent.

\section{B. Dependence of the beam heat load on filling patterns}

The dependence of the beam heat load as a function of current was studied for different filling patterns. ANKA can be filled with one bunch train consisting of 32 bunches with a bunch spacing of $2 \mathrm{~ns}$, or two or three bunch trains separated by $120 \mathrm{~ns}$. This allows to change the bunch current while the average current remains identical. Keeping in mind that for the following measurements less statistics is available, the beam heat load measured as a function of the average beam current for $16 \mathrm{~mm}$ gap (see Fig. 10) scales as

$$
P \propto \frac{I^{2}}{M},
$$

where $I$ is the stored average beam current and $M$ the total number of bunches per revolution. Such a scaling is typical for losses caused by resistive mechanisms.

In the inset of Fig. 10, the UHV pressure as a function of the average beam current is shown. The pressure rise is in some runs quite low and no peak is observed as a function of current. In case of electron bombardment a possible explanation would be the dependence of the peak current

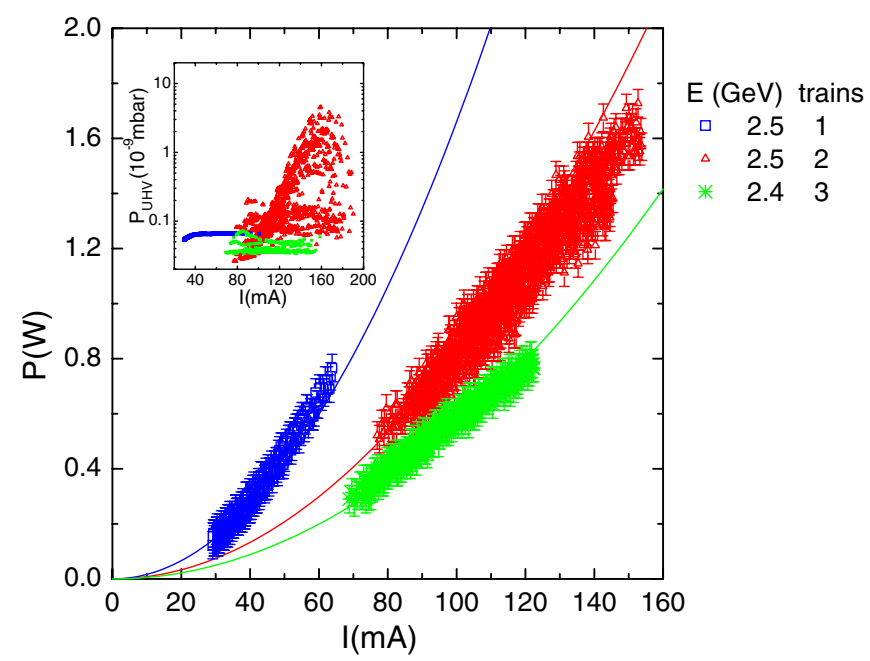

FIG. 10. (Color) The beam heat load reported as a function of the average beam current for three different filling patterns. The three curves are polynomial fits demonstrating the scaling law $P \propto I^{2} / M$. Blue line: $P(\mathrm{~W})=1.66 \times 10^{-4} I^{2}(\mathrm{~mA})$. Red line: $P(\mathrm{~W})=1.66 / 2 \times 10^{-4} I^{2}(\mathrm{~mA})$. Green line: $P(\mathrm{~W})=1.66 / 3 \times$ $10^{-4} I^{2}(\mathrm{~mA})$. In the inset the UHV pressure is shown as a function of average beam current. on the beam history: it is in fact known that the desorption coefficients and the secondary emission yields of the different gas molecules adsorbed on the vacuum chamber decrease with beam exposure time (electron dose) [15].

\section{Beam heat load with subpicosecond pulses}

In order to produce short bunches of a few hundreds femtoseconds ANKA can be operated in the so-called low momentum compaction mode [19]. The measured beam heat load for a run in the low momentum compaction mode with one train at $E=1.3 \mathrm{GeV}$ and a gap $=29 \mathrm{~mm}$ is shown in Fig. 11. The data can be well fitted by the resistive wall heating model using Eq. (2) taking into account the anomalous skin effect, assuming a RRR $=100$ and a bunch length of $500 \mathrm{fs}$. The value of $500 \mathrm{fs}$ is consistent with the bunch lengths measured in the low momentum compaction mode by means of $\mathrm{THz}$ edge synchrotron radiation [21]. During standard operation the bunch length varies with beam energy. The heat load induced by resistive wall effects should be higher at lower energies since the bunch length is shorter. This is not the case. So resistive wall heating seems to be dominant for short bunches but not for longer ones. We conclude that another heating mechanism must be responsible for the beam heat load observed during user operation.

\section{Heat load by changing the gas content in the undulator}

With the aim to see if the effect is caused by the gas composition, $\mathrm{H}_{2}$ was injected in the warm side before and

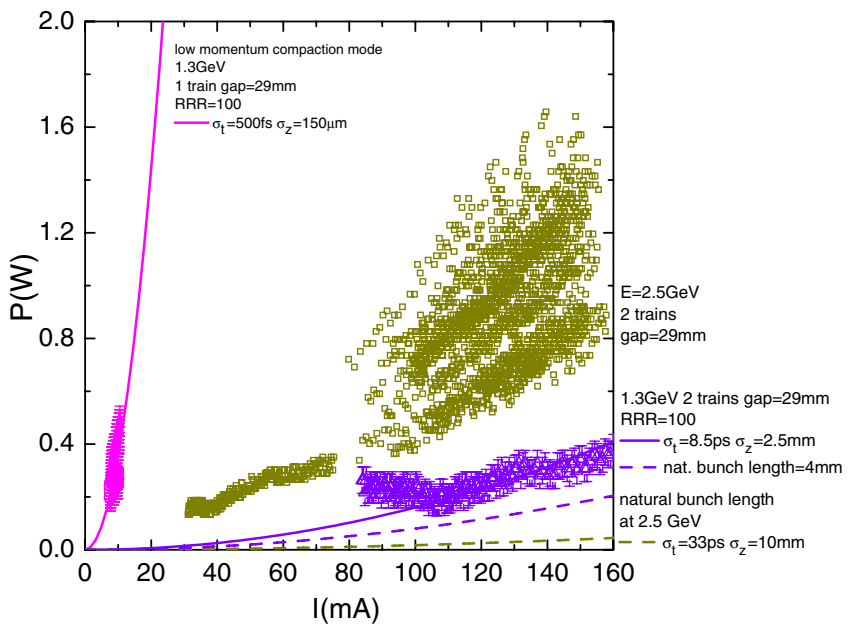

FIG. 11. (Color) The beam heat load reported as a function of the average beam current for three different bunch lengths. The four curves are theoretical predictions based on Eq. (2) computed for different bunch lengths as indicated. The dashed curves are obtained with the natural bunch length (defined only for the normal optics of the ring, so for longer bunches), derived from Ref. [20]. The theoretical curves are obtained by using bunch lengths values to fit the experimental data. 


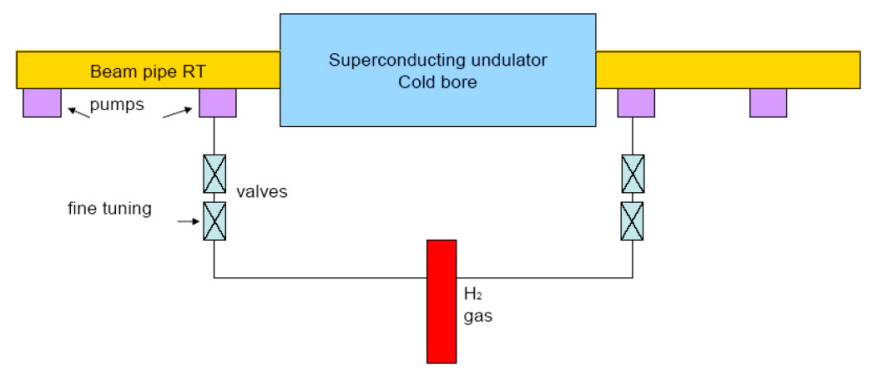

FIG. 12. (Color) Scheme of the experimental setup for $\mathrm{H}_{2}$ injection in the undulator.

after the undulator. A scheme of the experimental setup is shown in Fig. 12. The idea is to increase the equilibrium pressure to enhance the $\mathrm{H}_{2}$ surface coverage $s$ and consequently the beam heat load. We were able to change the equilibrium pressure only by about a factor of 2.5 from $2 \times$ $10^{-11}$ mbar to $5 \times 10^{-11} \mathrm{mbar}$, so the surface coverage could not significantly be changed (see Fig. 5). The results are shown in Fig. 13: the beam heat load does not change. Two runs with, respectively, three and two trains have been performed. Figure 13 also shows two successive runs without further injecting $\mathrm{H}_{2}$ and with pumps once on and once off. The pressure increases throughout these runs and the beam current where the peak pressure is observed shifts to lower values than before $\mathrm{H}_{2}$ injection (see the run with $E=2.5 \mathrm{GeV}$ with two trains, red circles). No significant difference is observed in the fills with/without $\mathrm{H}_{2}$ and with pumps on/off. As shown in the inset of the upper plot of Fig. 13, again a correlation is observed between the beam heat load and the pressure. We also observe (see lower plot of Fig. 13) that the current where the peak pressure is observed depends on the number of bunches: for two trains it is lower than for three trains.

\section{E. Pressure rise and heat load due to electron bombardment}

The equations of gas dynamic balance inside a vacuum chamber can be written as (see Refs. [22,23] and references therein)

$V \frac{d n}{d t}=q+q^{\prime}(s)-\alpha S\left(n-n_{e}(s, T)\right)+u \frac{d^{2} n}{d z^{2}}$

$A \frac{d s}{d t}=\alpha S\left(n-n_{e}(s, T)\right)-q^{\prime}(s)$,

where $n$ is the volume gas density, $s$ the surface density of the cryosorbed gas, $V$ the vacuum chamber volume, $A$ the vacuum chamber wall area, $q$ is the primary beam induced desorption flux, $q^{\prime}$ the secondary beam induced desorption flux (desorption of cryosorbed molecules), $\alpha$ the sticking coefficient, $S=A \bar{\nu} / 4$ is the ideal wall pumping speed, $\bar{\nu}$ is the mean molecular speed, $n_{e}$ the thermal equilibrium gas density, and $u$ the specific vacuum chamber conductance per unit axial length. The beam induced desorption flux
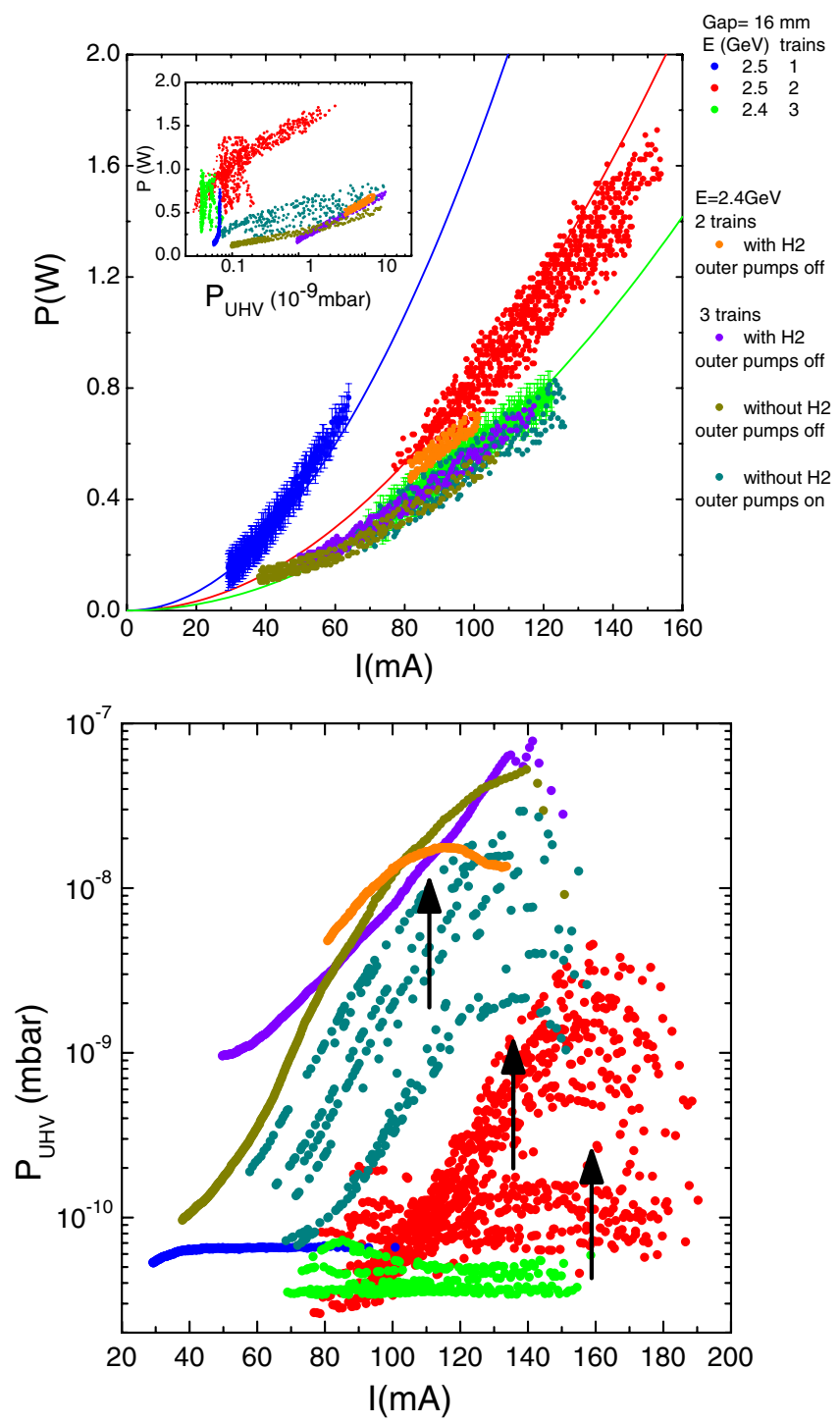

FIG. 13. (Color) The beam heat load (upper plot) and the UHV pressure (lower plot) are shown as a function of the average beam current $I$. In the upper plot the three curves (continuous lines) have been explained in Fig. 10. The arrows in the lower plot indicate the current at which the pressure has a peak. Starting from high currents they refer to: run with $E=$ $2.5 \mathrm{GeV}$, two trains and no $\mathrm{H}_{2}$ injected (red circles), run with $E=2.4 \mathrm{GeV}$, three trains, $\mathrm{H}_{2}$ injected and after injection (violet circles, dark yellow circles, and dark cyan circles), run with $E=$ $2.4 \mathrm{GeV}$, two trains and $\mathrm{H}_{2}$ injected (orange circles). In the inset the beam heat load is reported as a function of the UHV pressure. The beam parameters and the undulator gap are indicated in the upper plot for the different runs. In all cases the current in the superconducting coils of the undulator is zero.

consists of photon (PSD) and electron stimulated desorption:

$$
q=\eta \dot{\Gamma}+\phi \dot{\Theta} ; \quad q^{\prime}=\eta^{\prime} \dot{\Gamma}+\phi^{\prime} \dot{\Theta},
$$

where $\eta$ and $\eta^{\prime}$ are the primary and secondary electron 
stimulated desorption yields, $\dot{\Gamma}$ is the electron flux, $\phi$ and $\phi^{\prime}$ are the primary and secondary photodesorption yields, and $\dot{\Theta}$ is the photon flux.

The volume gas density $n$ at a temperature $T$ is related to the pressure measured at room temperature by

$$
n=\frac{P}{k_{B} \sqrt{T T_{\mathrm{RT}}}} .
$$

When the pressure reaches the maximum (see Fig. 7) as a function of time, $d n / d t=0$. The specific vacuum chamber conductance per unit axial length is given by $u=A_{c} D$, where $D=2 / 3 A_{c} \bar{\nu}$ is the Knudsen diffusion coefficient being $A_{c}$ the area of the rectangular cross section of the vacuum chamber. Axial diffusion can be neglected when $D A_{c} / L^{2}<S \alpha$ [22], which means

$$
\frac{8}{3} \frac{A_{c}^{2}}{A L^{2}}<\alpha
$$

Experimental values of the sticking coefficient for $\mathrm{H}_{2}$ at $4.2 \mathrm{~K}$ indicate $\alpha>0.02$ [24]. Even considering $\alpha=$ 0.02 condition (12) is satisfied for the geometry of the undulator vacuum chamber where $L=1.4 \mathrm{~m}$ and for a gap of $29 \mathrm{~mm}, A_{c}=0.00191 \mathrm{~m}^{2}$ and $A=0.266 \mathrm{~m}^{2}$. Neglecting axial diffusion $u d^{2} n / d z^{2} \approx 0$, the sum of the primary and recycling desorption yield of all gas species can be computed using the following equation:

$$
\frac{q+q^{\prime}}{\alpha}=S\left(n-n_{e}(s, T)\right)=S G \Delta P,
$$

where $\Delta P=P_{\max }-P_{e}$ with $P_{e} \lesssim 2 \times 10^{-11}$ mbar the thermal equilibrium pressure and

$$
G=\frac{1}{k_{B} \sqrt{T T_{\mathrm{RT}}}}=2 \times 10^{17} \mathrm{~cm}^{-3} \text { mbar. }
$$

For the ANKA cold bore vacuum chamber with gap $=$ $29 \mathrm{~mm}$ and average beam current $I=100 \mathrm{~mA}$, the photon flux impinging on the lower and upper surfaces is $\dot{\Theta} \approx$ $10^{16}$ photons/s.

If the heat load observed is generated by electron bombardment and assuming a mean electron energy $\Delta W=$ $10 \mathrm{eV}$ (for a typical $3.6 \times 10^{9}$ electrons/bunch), the estimated electron flux for a heat load of $P=1 \mathrm{~W}$ is $\dot{\Gamma} \approx$ $6 \times 10^{17}$ electrons/s. Being $\phi+\phi^{\prime} \lesssim \eta+\eta^{\prime} \quad[15,25]$, we can neglect the PSD to the beam desorption flux, so that $q=\eta \dot{\Gamma}$ and $q^{\prime}=\eta^{\prime} \dot{\Gamma}$. The observed $\Delta P$ ranges from $2 \times 10^{-11}$ mbar to $8 \times 10^{-8}$ mbar. For $\mathrm{H}_{2}$ the mean molecular speed at $4.2 \mathrm{~K}$ is $\bar{\nu}=210 \mathrm{~m} / \mathrm{s}$. The area of the vacuum chamber for a gap $=29 \mathrm{~mm}$ is $A=$ $0.266 \mathrm{~m}^{2}$. Applying Eq. (13), we find that the sum of the primary and secondary desorption yields for $\mathrm{H}_{2}(\eta+$ $\left.\eta^{\prime}\right) / \alpha$ ranges between $10^{-4}$ molecules/electron to 4 molecules/electron. Our values are in good agreement with the ones measured at COLDEX [15] that range between $10^{-2}$ molecules/electron for an electron dose of
$2 \times 10^{19}$ electrons $/ \mathrm{cm}^{2}$ to 30 molecules/electron for an electron dose of $10^{17}$ electrons $/ \mathrm{cm}^{2}$, considering that in our case the temperature is lower $(4.2 \mathrm{~K}$ instead of $12 \mathrm{~K})$, the mean electron energy is an order of magnitude smaller ( $10 \mathrm{eV}$ instead of $100 \mathrm{eV} \mathrm{[15])} \mathrm{and} \mathrm{that} \mathrm{our} \mathrm{electron} \mathrm{dose} \mathrm{is}$ in some cases much higher (after two weeks of normal user operation it is about $2 \times 10^{20}$ electrons $/ \mathrm{cm}^{2}$ ).

\section{CONCLUSIONS}

The measured beam heat load has been compared with theoretical predictions from different models. Synchrotron radiation cannot explain the data since it predicts a linear dependence with current which is not observed. The resistive wall heating model can fit the data for short bunches but it does not for longer bunches. The observed heat load scales with $I^{2} / M$, which is typical for resistive effects. On the other hand, a smaller beam heat load is found at lower beam energies when the bunch length is shorter. Resistive effects would imply higher beam heat load for shorter bunches. Resistive effects also cannot explain the large variation in the beam heat load.

A nonlinear pressure rise with current is observed. This rise might be due to $\mathrm{H}_{2}$ recycling and/or electron multipacting. The average beam current where the pressure assumes a maximum is lower when the pressure is higher and it decreases also for higher bunch currents. The pressure does not always peak at a certain value of beam current. One possible explanation would be the dependence of the surface properties on the beam history: it is in fact known that the desorption coefficients and the secondary emission yields of the different gas molecules adsorbed on the vacuum chamber decrease by increasing the beam exposure time [15].

A simple model of electron bombardment appears to be consistent with the beam heat load and pressure rise observed during normal user operation (longer bunches). Assuming this model is valid, we still need to understand the mechanism responsible for releasing the electrons from the gas layer cryosorbed on the wall of the vacuum chamber.

\section{ACKNOWLEDGMENTS}

The authors are indebted to V. Baglin (CERN), O. B. Malyshev (ASTeC, Daresbury), and E. Wallén (MAX-Lab, Lund) for valuable discussions. The measurements at ANKA were performed together with the ANKA machine group: I. Birkel, E. Huttel, A.-S. Müller, and P. Wesolowski. The authors would like to thank E. Huttel for useful discussions and A. Völker for technical support in setting up the $\mathrm{H}_{2}$ experiment.

[1] J. Chavanne and P. Elleaume, Proceedings of EPAC 2006, Edinburgh, Scotland, 2006. 
[2] S. Casalbuoni et al., Phys. Rev. ST Accel. Beams 9, 010702 (2006).

[3] E. Levitchev et al., Proceedings of EPAC 2006, Edinburgh, Scotland, 2006.

[4] J. A. Clarke et al., Proceedings of EPAC 2006, Edinburgh, Scotland, 2006.

[5] http://ankaweb.fzk.de/

[6] S. Chouhan et al., Proceedings of PAC 2003, Portland, Oregon, USA (IEEE, Piscataway, NJ, 2003).

[7] SHI Cryogenics Group, http://www.apdcryogenics.com.

[8] S. Casalbuoni et al., Proceedings of EPAC 2006, Edinburgh, Scotland, 2006.

[9] E. Wallén and G. LeBlanc, Cryogenics 44, 879 (2004).

[10] W. Chou and F. Ruggiero, LHC Project Note 2 (SL/AP), 1995.

[11] H. London, Proc. R. Soc. A 176, 522 (1940); A. B. Pippard, Proc. R. Soc. A 191, 385 (1947); G. E. H. Reuter and E. H. Sondheimer, Proc. R. Soc. A 195, 336 (1948); R. G. Chambers, Proc. R. Soc. A 215, 481 (1952).

[12] N. Ashcroft and D. Mermin, Solid State Physics (Holt, Rinehart and Winston, New York, 1976).

[13] O. Gröbner, Proceedings of PAC 1997, Vancouver, Canada (IEEE, Piscataway, NJ, 1997).

[14] http://cas.web.cern.ch/cas/Spain-2006/PDFs/Hilleret-1. pdf.
[15] V. Baglin and B. Jenninger, LHC Project Report 721, 2004.

[16] E. Wallén, J. Vac. Sci. Technol. 14, 2916 (1996).

[17] The pressure values reported in this paper are measured at room temperature. The corresponding values at low temperature can be obtained applying the Knudsen relation $P(T)=(T[\mathrm{~K}] / 300 \mathrm{~K})^{1 / 2} P(300 \mathrm{~K})$.

[18] A. Kulikov et al., Proceedings of PAC 2001, Chicago, Illinois (IEEE, Piscataway, NJ, 2001).

[19] A.-S. Müller et al., Proceedings of PAC 2005, Knoxville, Tennessee, USA (IEEE, Piscataway, NJ, 2005).

[20] F. Perez et al., Proceedings of PAC 2003, Portland, Oregon, USA (IEEE, Piscataway, NJ, 2003).

[21] A. Bernhard et al., Proceedings of Applied Superconductivity Conference, Seattle, WA, USA, 2006 [IEEE Trans. Appl. Supercond. 17, No. 2 (2007)].

[22] W. C. Turner, Proceedings of PAC 1993, Washington, D.C. (IEEE, Piscataway, NJ, 1993).

[23] I. R. Collins and O. B. Malyshev, LHC Project Note 274, 2001.

[24] S. Andersson, L. Wilzén, M. Persson, and J. Harris, Phys. Rev. B 40, 8146 (1989); V. V. Anashin et al., J. Vac. Sci. Technol. 12, 1663 (1994).

[25] V. V. Anashin et al., Vacuum 48, 785 (1997). 